\title{
No pain, if you've got game
}

\author{
Allowing children to engage in "active" distraction \\ techniques—such as playing a video game-during \\ venipuncture can lead to reduced pain and anxiety.
}

\section{PRACTICE CHANGER}

Employ active distraction, such as playing a video game, rather than passive distraction (eg, watching a video) to reduce pain and anxiety during pediatric venipuncture.

\section{STRENGTH OF RECOMMENDATION}

B: Based on a single, high-quality, randomized controlled trial (RCT). ${ }^{1}$

Inan $\mathrm{G}$, Inal S. The impact of 3 different distraction techniques on the pain and anxiety levels of children during venipuncture: a clinical trial. Clin J Pain. 2019;35:140-147.

\section{ILLUSTRATIVE CASE}

An 8-year-old girl with congenital heart disease (status: post repair) arrives at your clinic for a routine appointment. Since the age of 12 months, she has experienced significant anxiety during medical visits, especially with blood draws and injections. She enjoys playing video games on her new tablet computer. Her parents want to know what you can do to reduce her anxiety and pain during today's scheduled blood draw. Should you recommend that she continue playing video games during the venipuncture?

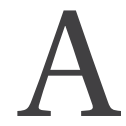

dequately managing pain while performing venipuncture in children can improve the quality of the experience, reduce children's fear of going to the doctor, and increase efficiency in medical practice. ${ }^{2}$ Since pharmacologic pain-control methods may have adverse effects, distraction techniques-engaging the child in another activity during a procedure-are commonly used instead to help reduce a child's pain. These techniques can be active or passive.
Studies have demonstrated that both active and passive distraction techniques reduce children's pain during medical procedures, including venipuncture. Passive techniques, such as nurse coaching ${ }^{3}$ and watching cartoons, ${ }^{4}$ have been found to reduce distress and pain. Active distraction techniques, such as playing video games while undergoing a painful procedure (eg, dressing a wound), have been shown to be more effective than passive techniques. ${ }^{5,6}$

A Cochrane review and meta-analysis of distraction and hypnosis for needle-related pain and distress in children demonstrated reduced pain, but the quality of evidence was low and the review recommended improved methodological rigor and trial reporting. ${ }^{7}$ Another systematic review and analysis showed strong support for distraction for reducing pain; however, the quality of evidence was low and the researchers cited problems with characteristics of the distraction interventions, child age, and risk of bias in the studies. ${ }^{8}$

There has been a lack of RCTs comparing the effectiveness and superiority of active vs passive distraction techniques. The first highquality RCT to directly compare 3 of the most common distraction techniques to a control group was recently conducted in a large training and research hospital in Turkey. ${ }^{1}$

\section{STUDY SUMMARY}

Pain and anxiety levels were lowest in actively distracted children

The RCT included 180 children ages 6 to 10 years randomly assigned to 1 of 3 intervention groups
Benjamin J. McCollum, MD; Stephen J. Conner, MD; J. Scott Earwood, MD

Family Medicine Residency Program, Eisenhower Army Medical Center,

Fort Gordon, GA

DEPUTY EDITOR Katherine Hale, PharmD, BCPS, MFA

Family Physicians Inquiries Network

doi: $10.12788 /$ fp. 0132 


\section{$>$}

\section{Allow children} to play a video game during procedures such as venipuncture; doing so reduces pain and anxiety. or a control group. ${ }^{1}$ Phlebotomy was performed while children watched a cartoon, played a video game, were distracted by parental interaction, or had no distraction (control group).

Investigators independently measured pain and anxiety in the patient and perceived pain and anxiety according to both a family member and a health care worker (medical observer). Researchers used the previously validated Children's Fear Scale and the Wong-Baker Pain Scale.,10 The Children's Fear Scale was used to assess anxiety in children on a scale of 0 (picture of a calm face) to 4 (picture of the most fearful face). The WongBaker Pain Scale was used to assess pain on a scale of 0 (no hurt: happy face) to 10 (hurts worst: saddest face).

Results. The pain and anxiety scores were significantly lower in all of the intervention groups compared with the control group $(P<.05)$. The video game (active distraction) group had the lowest levels of both pain and anxiety. The self-reported Children's Fear Scale scores of children in the video game group were 0.27 , compared with 0.76 in the cartoon group, 1.24 in the parental distraction group, and 2.22 in the control group. The anxiety scores recorded by the family member and the medical observer showed similar significant differences.

The Wong-Baker Pain Scale scores showed similar differences in self-reported pain for the video game group (1.42) compared with the cartoon group (3.02), the parental distraction group (2.89), and the control group (5.11). Pain scores reported by the family member and the medical observer (respectively) also reflected benefit from any type of distraction, with active gameplaying as the most effective type of distraction (video game: 1.69 and 1.96; cartoon: 3.07 and 3.20; parental distraction: 3.56 and 4.22; and control: 5.29 and 6.13).

In addition, the intraclass correlation coefficient was 0.67 to $0.924(P<.01)$, suggesting that the reports from the child, parent, and medical observer about the child's pain and anxiety were highly correlated.

\section{WHAT'S NEW}

\section{All distraction techniques provide} benefit, but there's a clear winner In this RCT of children undergoing phle- botomy, both active and passive distraction techniques were superior to no distraction in terms of perceived pain and anxiety by the child, a health care provider, or a parent. The active-distraction group played a video game, while the passive-distraction groups watched a cartoon or interacted with a parent. Active distraction was superior to passive distraction.

\section{CAVEATS}

\section{Procedure time was short; intervention not blinded}

One potential weakness of this study is that it was not a double-blinded trial. Blinding was not possible for much of the study as the patient, parent, and medical observer were fully aware of the intervention or lack thereof. However, the parent and medical observer were blinded to each other's assessments of the child's pain and anxiety.

Furthermore, the study was conducted at a single institution in Turkey. There could be cultural differences in reporting of pain and anxiety compared to Western cultures.

Finally, the average duration of the procedure in this study was 3 minutes, with a range of 1 to 5 minutes. It is unclear if the findings can be extrapolated to more timeconsuming procedures.

\section{CHALLENGES TO IMPLEMENTATION}

\section{Technology is not available to all}

The use of tablet computers may seem increasingly ubiquitous, but not all families have access to these devices. Another challenge is that phlebotomy/clinic personnel must learn to work around the device. JFP

\section{ACKNOWLEDGEMENT}

The PURLs Surveillance System was supported in part by Grant Number UL1RR024999 from the National Center for Research Resources, a Clinical Translational Science Award to the University of Chicago. The content is solely the responsibility of the authors and does not necessarily represent the official views of the National Center for Research Resources or the National Institutes of Health. Copyright @ 2021 . The Family Physicians Inquiries Network. All rights reserved.

\footnotetext{
References

1. Inan G, Inal S. The impact of 3 different distraction techniques on the pain and anxiety levels of children during venipuncture: a clinical trial. Clin J Pain. 2019;35:140-147.
} 
2. Fein JA, Zempsky WT, Cravero JP, Committee on Pediatric Emergency Medicine and Section on Anesthesiology and Pain Medicine; American Academy of Pediatrics. Relief of pain and anxiety in pediatric patients in emergency medical systems. Pediatrics. 2012;130:e1391-e1405.

3. Cohen LL, Blount RL, Panopoulos G. Nurse coaching and cartoon distraction: an effective and practical intervention to reduce child, parent, and nurse distress during immunizations. J Pediatr Psychol. 1997;22:355-370.

4. Downey VA, Zun LS. The impact of watching cartoons for distraction during painful procedures in the emergency department. Pediatr Emerg. 2012;28:1033-1035.

5. Hussein $\mathrm{H}$. Effect of active and passive distraction on decreasing pain associated with painful medical procedures among school aged children. World J Nurs Sci. 2015;1:13-23.

6. Nilsson S, Enskär K, Hallqvist C, et al. Active and passive dis- traction in children undergoing wound dressing. J Pediatr Nurs. 2013;28:158-166.

7. Birnie KA, Noel M, Chambers CT, et al. Psychological interventions for needle-related procedural pain and distress in children and adolescents. Cochrane Database Syst Rev. 2018;10: CD005179.

8. Birnie KA, Noel M, Parker JA, et al. Systematic review and meta-analysis of distraction and hypnosis for needle-related pain and distress in children and adolescents. J Pediatr Psychol. 2014;39:783-808.

9. McMurtry CM, Noel M, Chambers CT, et al. Children's fear during procedural pain: preliminary investigation of the Children's Fear Scale. Health Psychol. 2011;30:780-788.

10. Wong DL, Baker CM. Pain in children: comparison of assessment scales. Pediatric Nurs. 1988;14:9-17. 